CARDIOVASCULAR MEDICINE

\title{
Reduced between-hospital variation in short term survival after acute myocardial infarction: the result of improved cardiac care?
}

\author{
N F Murphy, K MacIntyre, S Stewart, S Capewell, J J V McMurray
}

Heart 2005;91:726-730. doi: 10.1136/hrt.2004.042929

See end of article for authors' affiliations

\section{Correspondence to:} Professor John McMurray, Department of Cardiology, Western Infirmary, Glasgow G11 6NT, UK; j.mcmurray@bio.gla.ac.uk

Accepted 26 August 2004
Objectives: To re-examine interhospital variation in 30 day survival after acute myocardial infarction (AMI) 10 years on to see whether the appointment of new cardiologists and their involvement in emergency care has improved outcome after AMI.

Design: Retrospective cohort study.

Setting: Acute hospitals in Scotland.

Participants: 61484 patients with a first AMl over two time periods: 1988-1991; and 1998-2001.

Main outcome measures: 30 day survival.

Results: Between 1988 and 1991, median 30 day survival was $79.2 \%$ (interhospital range 72.1-85.1\%).

The difference between highest and lowest was 13.0 percentage points lage and sex adjusted, 12.1 percentage points). Between 1998 and 2001, median survival rose to $81.6 \%$ (and range decreased to 78.0-85.6\%) with a difference of 7.6 (adjusted 8.8) percentage points. Admission hospital was an independent predictor of outcome at 30 days during the two time periods ( $p<0.001$ ). Over the period 1988-1991, the odds ratio for death ranged, between hospitals, from 0.71 (95\% confidence interval (Cl) 0.58 to 0.88$)$ to $1.50(95 \% \mathrm{Cl} 1.19$ to 1.89$)$ and for the period $1998-2001$ from $0.82(95 \% \mathrm{Cl} 0.60$ to $1.13)$ to 1.46 (95\% Cl 1.07 to 1.99$)$. The adjusted risk of death was significantly higher than average in nine of 26 hospitals between 1988 and 1991 but in only two hospitals between 1998 and 2001.

Conclusions: The average 30 day case fatality rate after admission with an AMI has fallen substantially over the past 10 years in Scotland. Between-hospital variation is also considerably less notable because of better survival in the previously poorly performing hospitals. This suggests that the greater involvement of cardiologists in the management of AMI has paid dividends. in a study of the period 1988 to 1991, substantial variation in 30 day survival after admission with acute myocardial infarction (AMI) was identified between hospitals in Scotland. ${ }^{1}$ Since that study, at least one cardiologist has been appointed in each Scottish hospital and acute medical care has been reorganised in most to ensure more rapid and appropriate care of patients suspected to have this diagnosis. ${ }^{2}$ We have re-examined variation in survival after admission with AMI 10 years after the initial study. Our aim was to determine whether there has been a reduction in the variation in survival between Scottish hospitals in this more recent time period compared with the earlier one.

\section{METHODS}

\section{Data sources}

A Scotland-wide retrospective study was carried out by using the linked Scottish Morbidity Record (SMR) database. ${ }^{3}$ Data on all National Health Service (NHS) hospital discharges in Scotland (total population about 5.1 million) are collected (on the SMR 1 form) and collated by the Information and Statistics Division (ISD) of the NHS. Linking records through probability matching (with an accuracy of about 98\%) permits analysis of data at the level of the individual patient rather than the episode of care. This database is also linked to that of the Registrar General for death certificates (also with an estimated accuracy of 98\%). For any given patient, therefore, readmission after an index hospitalisation can be tracked (and the nature of the readmission identified). Patients' deaths are also recorded. ${ }^{3}$

We identified all patients discharged (alive or dead) from Scottish hospitals during two periods of time-between
October 1988 and September 1991, and between October 1998 and September 2001 - with a principal diagnosis of AMI (International classification of diseases (ICD), ninth revision code 410 and ICD-10 codes I21 and I22). ${ }^{4}$ Only discharges for a "first" AMI were analysed. A first AMI was defined as one where the patient had no prior hospital discharge for AMI. Only hospitals admitting patients with emergency medical problems during both study periods $(n=26)$ were compared in this analysis.

\section{Quality of data}

The Quality Assessment and Accreditation Unit of ISD routinely validates a $1 \%$ sample of SMR 1 records (where SMR I data are compared with those in each patient's medical record). The accuracy of AMI coded as a principal diagnosis has been shown to be $97 \% .^{5}$ The accuracy of coding secondary conditions is $75 \%{ }^{6}$

\section{Baseline data}

Each patient's SMR 1 record provided information on age, sex, postcode sector, date of discharge, hospital discharge from, previous discharges, and date of death if it occurred. Postcodes of residence were used to assign a Carstairs deprivation category ranging from 1 (least deprived) to 5 (most deprived) to each patient. ${ }^{7}$ These categories are derived from the 1991 census data on the proportion of residents who

Abbreviations: $\mathrm{AMI}$, acute myocardial infarction; $\mathrm{Cl}$, confidence interval; ICD, International classification of diseases; ISD, Information and Statistics Division; NHS, National Health Service; SMR, Scottish Morbidity Record 
are unemployed, live in overcrowded accommodation, do not have a car, or belong to a low occupational social class. There is a strong concordance between Carstairs scores for postcode sectors in 1991 and 2001 (Pearson's correlation coefficient $r=0.955){ }^{8}$

All prior discharges within five years were identified by retrospective linkage. Secondary conditions on the index admission allowed the identification of up to five comorbidities.

\section{Statistical analysis}

Baseline characteristics of patients admitted to hospital within the two time periods were compared by $\chi^{2}$ test for discrete variables and Student's $t$ test for continuously distributed variables. Crude 30 day survival was calculated for each hospital and for Scotland as a whole by use of the actuarial life table method. Indirect standardisation was then used to adjust hospital specific 30 day survival rates for age and sex differences between the hospital population and the whole Scottish population. Multiple logistic regression models were used to determine the independent effect of hospital on 30 day case fatality after adjusting for the effects of age, sex, deprivation category, and prior and concurrent morbidities. The hospitals were entered into the logistic models as one categorical variable. The "index" hospital was set as that with a case fatality nearest the Scottish crude case fatality for that time period. All variables were entered simultaneously into the models. Each model was subject to the Hosmer-Lemeshow goodness of fit test, and all were nonsignificant. All analyses were undertaken with use of SPSS version 11 (SPSS Inc, Chicago, Illinois, USA).

\section{RESULTS}

\section{Baseline characteristics}

Table 1 shows the baseline characteristics of the 61484 patients discharged with a first AMI during the two time periods of study: $36108(58.7 \%)$ were admitted between 1988 and 1991 and 25376 (41.3\%) were admitted between 1998 and 2001. The mean age of men and women increased between these time periods $(\mathrm{p}<0.001$ ). In the period $1988-$ $1991,48.2 \%$ of men were aged 65 or over. This proportion rose to $50.5 \%$ in $1998-2001$ ( $\mathrm{p}<0.001)$. The corresponding figures for women were $72.1 \%$ and $75.4 \%(p<0.001)$, respectively. Between 1988 and 1991, 39.4\% of men and $45.3 \%$ of women had one or more co-morbidities or prior admissions. This proportion rose to $46.9 \%$ in men and $52.9 \%$ in women in 1998-2001 $(\mathrm{p}<0.001)$.

\section{Thirty day survival after hospital admission}

Table 2 shows improved crude and age and sex adjusted 30 day survival. Crude 30 day survival for first AMI increased from $77.7 \%$ in $1988-1991$ to $80.9 \%$ in $1998-2001$ in Scotland as a whole. The number of hospitals with a 30 day survival of less than 75\% decreased from five to none (and those with a survival of less than $80 \%$ from 18 to seven) between these two periods.

Table 2 and fig 1 show that variation in crude and age and sex adjusted 30 day survival decreased. Between 1988 and 1991 the median crude 30 day survival rate was $79.2 \%$ with a range from $72.1 \%$ to $85.1 \%$ across hospitals, a difference of 13.0 percentage points. After standardising for age and sex, there was still a difference of 12.1 percentage points in 30 day survival between hospitals.

Between 1998 and 2001 the median crude 30 day survival rate was $81.6 \%$ with a range from $78.0 \%$ to $85.6 \%$. This difference of 7.6 percentage points increased slightly to 8.8 percentage points after standardising for age and sex. Therefore, the interhospital variability decreased between these two time periods after adjustment for age and sex differences. The hospitals with the worst survival in 19881991 improved the most.

\section{Multiple logistic regression 1988-1991}

After adjustment for age, sex, deprivation category, and prior (previous admissions) and concurrent morbidities (secondary discharge diagnoses), admission hospital was a highly significant predictor of outcome at 30 days $(p<0.001)$.

Table 1 Baseline characteristics of patients discharged after a first acute myocardial infarction during the periods 1998-2001 and 1988-1991

\begin{tabular}{|c|c|c|c|c|}
\hline & \multicolumn{2}{|c|}{$1988-1991(n=36108)$} & \multicolumn{2}{|c|}{$1998-2001(n=25376)$} \\
\hline & Men & Women & Men & Women \\
\hline Total 1998-2001 & $20985(58.1 \%)$ & $15123(41.9 \%)$ & $14953(58.9 \%)$ & $10423(41.1 \%)$ \\
\hline Age (years)* & $64.4(65.0)$ & $71.6(73.0)$ & $65.0(66.0)$ & $72.9(74.0)$ \\
\hline \multicolumn{5}{|l|}{ Age group (years) } \\
\hline$<55$ & $4892(23.3 \%)$ & $1378(9.1 \%)$ & $3677(24.6 \%)$ & $987(9.5 \%)$ \\
\hline $55-64$ & $5997(28.6 \%)$ & $2840(18.8 \%)$ & $3713(24.8 \%)$ & $1573(15.1 \%)$ \\
\hline $65-74$ & $6036(28.8 \%)$ & $4726(31.3 \%)$ & $4132(27.6 \%)$ & $3046(29.2 \%)$ \\
\hline $75-84$ & $3518(16.8 \%)$ & $4767(31.5 \%)$ & $2768(18.5 \%)$ & $3296(31.6 \%)$ \\
\hline$\geqslant 85$ & $542(2.6 \%)$ & $1412(9.3 \%)$ & $663(4.4 \%)$ & $1521(14.6 \%)$ \\
\hline \multicolumn{5}{|l|}{ Deprivation categories } \\
\hline I (least deprived) & $3416(16.3 \%)$ & $2325(15.4 \%)$ & $2665(17.8 \%)$ & $1837(17.6 \%)$ \\
\hline$\|$ & $3856(18.4 \%)$ & $2715(18.0 \%)$ & 2950 (19.7\%) & 1935 (18.6\%) \\
\hline III & 3850 (18.3\%) & $2784(18.4 \%)$ & $3057(20.4 \%)$ & $2108(20.2 \%)$ \\
\hline IV & $4156(19.8 \%)$ & $3096(20.5 \%)$ & $2927(19.6 \%)$ & $2146(20.6 \%)$ \\
\hline V (most deprived) & $4437(21.1 \%)$ & $3409(22.5 \%)$ & $2991(20.0 \%)$ & $2261(21.7 \%)$ \\
\hline Missing data & $1270(6.1 \%)$ & $794(5.3 \%)$ & $363(2.4 \%)$ & $136(1.3 \%)$ \\
\hline \multicolumn{5}{|c|}{ Prior admission or co-morbidity } \\
\hline CHD & $2187(10.4 \%)$ & $1577(10.4 \%)$ & $1297(8.7 \%)$ & $857(8.2 \%)$ \\
\hline Other heart disease & $4048(19.3 \%)$ & $3619(23.9 \%)$ & $3901(26.1 \%)$ & $3305(31.7 \%)$ \\
\hline Stroke & $743(5.0 \%)$ & $604(5.8 \%)$ & $909(4.3 \%)$ & $962(6.4 \%)$ \\
\hline Peripheral arterial disease & $850(5.7 \%)$ & $561(5.4 \%)$ & $825(3.9 \%)$ & $539(3.6 \%)$ \\
\hline Respiratory disease $\ddagger$ & $1154(5.5 \%)$ & $799(5.3 \%)$ & $1396(9.3 \%)$ & $1149(11.0 \%)$ \\
\hline Malignancy & $823(3.9 \%)$ & $559(3.7 \%)$ & $1038(6.9 \%)$ & $697(6.7 \%)$ \\
\hline Diabetes mellitus & $940(4.5 \%)$ & $907(6.0 \%)$ & $162(1.1 \%)$ & $160(1.5 \%)$ \\
\hline Any of above & $8274(39.4 \%)$ & $6851(45.3 \%)$ & $7018(46.9 \%)$ & $5510(52.9 \%)$ \\
\hline
\end{tabular}


Table 2 Crude and age and sex adjusted 30 day survival after admission with a first acute myocardial infarction in 19881991 and 1998-2001

\begin{tabular}{|c|c|c|c|c|c|c|}
\hline \multirow[b]{2}{*}{ Hospital } & \multicolumn{3}{|c|}{ Crude 30 day survival } & \multicolumn{3}{|c|}{ Age and sex adjusted 30 day survival } \\
\hline & $1988-1991$ & 1998-2001 & $\begin{array}{l}\text { Difference } \\
\text { (percentage points) }\end{array}$ & 1988-1991 & 1998-2001 & $\begin{array}{l}\text { Difference } \\
\text { (percentage points) }\end{array}$ \\
\hline All in Scotland & $36108(77.7 \%)$ & $25376(80.9 \%)$ & 3.2 & NA & NA & NA \\
\hline 1 & $1361(74.1 \%)$ & $939(85.5 \%)$ & 11.4 & $73.8 \%$ & $84.9 \%$ & 11.1 \\
\hline 2 & 1209 (76.8\%) & $1156(83.3 \%)$ & 6.5 & $76.1 \%$ & $84.1 \%$ & 8 \\
\hline 3 & $1095(78.2 \%)$ & $685(84.7 \%)$ & 6.5 & $78.4 \%$ & $84.1 \%$ & 5.7 \\
\hline 4 & 2309 (81.6\%) & $1651(83.4 \%)$ & 1.8 & $80.1 \%$ & $82.5 \%$ & 2.4 \\
\hline 5 & $2099(81.4 \%)$ & $2000(83.1 \%)$ & 1.7 & $80.0 \%$ & $83.3 \%$ & 3.3 \\
\hline 6 & $919(82.8 \%)$ & $714(85.6 \%)$ & 2.8 & $81.7 \%$ & $83.7 \%$ & 2 \\
\hline 7 & $922(83.8 \%)$ & 730 (81.4\%) & -2.4 & $83.1 \%$ & $82.7 \%$ & -0.4 \\
\hline 8 & $1948(84.5 \%)$ & $1365(82.8 \%)$ & -1.7 & $84.5 \%$ & $82.7 \%$ & -1.8 \\
\hline 9 & $601(80.5 \%)$ & $504(81.0 \%)$ & 0.5 & $81.3 \%$ & $81.9 \%$ & 0.6 \\
\hline 10 & $957(72.9 \%)$ & $586(81.4 \%)$ & 8.5 & $73.7 \%$ & $82.5 \%$ & 8.8 \\
\hline 11 & 912 (76.9\%) & $605(82.5 \%)$ & 5.6 & $75.8 \%$ & $82.3 \%$ & 6.5 \\
\hline 12 & $1173(80.8 \%)$ & $668(83.1 \%)$ & 2.3 & $79.3 \%$ & $80.6 \%$ & 1.3 \\
\hline 13 & $536(78.5 \%)$ & $452(82.1 \%)$ & 3.6 & $77.6 \%$ & $81.1 \%$ & 3.5 \\
\hline 14 & 1314 (82.0\%) & $1137(81.6 \%)$ & -0.4 & $79.0 \%$ & $81.7 \%$ & 2.7 \\
\hline 15 & $706(78.5 \%)$ & $861(81.7 \%)$ & 3.2 & $77.0 \%$ & $80.7 \%$ & 3.7 \\
\hline 16 & $664(73.6 \%)$ & $688(81.1 \%)$ & 7.5 & $73.9 \%$ & $80.7 \%$ & 6.8 \\
\hline 17 & $1029(79.3 \%)$ & $575(80.4 \%)$ & 1.1 & $79.3 \%$ & $80.7 \%$ & 1.4 \\
\hline 18 & $1013(77.7 \%)$ & $582(81.6 \%)$ & 3.9 & $77.7 \%$ & $80.3 \%$ & 2.6 \\
\hline 19 & $1243(80.8 \%)$ & $1093(80.1 \%)$ & -0.7 & $78.6 \%$ & $79.4 \%$ & 0.8 \\
\hline 20 & $470(80.6 \%)$ & $419(78.0 \%)$ & -2.6 & $82.1 \%$ & $80.6 \%$ & -1.5 \\
\hline 21 & $1188(72.1 \%)$ & $837(80.1 \%)$ & 8 & $72.4 \%$ & $78.9 \%$ & 6.5 \\
\hline 22 & $598(85.1 \%)$ & 761 (82.4\%) & -2.7 & $82.7 \%$ & $79.1 \%$ & -3.6 \\
\hline 23 & 839 (79.0\%) & $815(80.5 \%)$ & 1.5 & $76.5 \%$ & $78.5 \%$ & 2 \\
\hline 24 & $1020(78.2 \%)$ & $1106(81.0 \%)$ & 2.8 & $74.0 \%$ & $78.2 \%$ & 4.2 \\
\hline 25 & 1201 (77.3\%) & $799(79.2 \%)$ & 1.9 & $75.4 \%$ & $78.1 \%$ & 2.7 \\
\hline 26 & $1455(80.8 \%)$ & $805(78.5 \%)$ & -2.3 & $78.6 \%$ & $76.1 \%$ & -2.5 \\
\hline Total $^{*}$ & $28781(79.2 \%)$ & $22533(81.6 \%)$ & 2.4 & $78.6 \%$ & $80.9 \%$ & 2.3 \\
\hline
\end{tabular}

The odds ratio for death after admission with AMI ranged from 0.71 (95\% confidence interval (CI) 0.58 to 0.88 ) to 1.50 (95\% CI 1.19 to 1.89 ) between hospitals. The index hospital was hospital 18, which had the same crude survival as Scotland as a whole (fig 2A). The adjusted risk of death within 30 days after an admission with AMI was significantly higher than average in nine (and better in one) of 26 hospitals.

\section{8-2001}

Admission hospital remained an independent predictor of outcome at 30 days $(\mathrm{p}<0.001)$. The odds ratio for death ranged from 0.82 (95\% CI 0.60 to 1.13 ) to 1.46 (95\% CI 1.07 to 1.99 ) between hospitals relative to hospital 9, which had a crude survival similar to that of Scotland as a whole (fig 2B). Interhospital variation was less and the adjusted risk of death was significantly higher than average in only two hospitals.

\section{DISCUSSION}

There are two notable findings of this study. Firstly, despite an increase in age and documented co-morbidity, patients with AMI had a higher 30 day survival in Scotland between 1998 and 2001 than in the period 1988 to 1991. Secondly, and most germane to the aims of this study, interhospital variation has declined substantially between 1988 and 1991 and between 1998 and 2001.

Though not the principal aim of this study, it was of interest to be able to confirm that there has been a fall in the

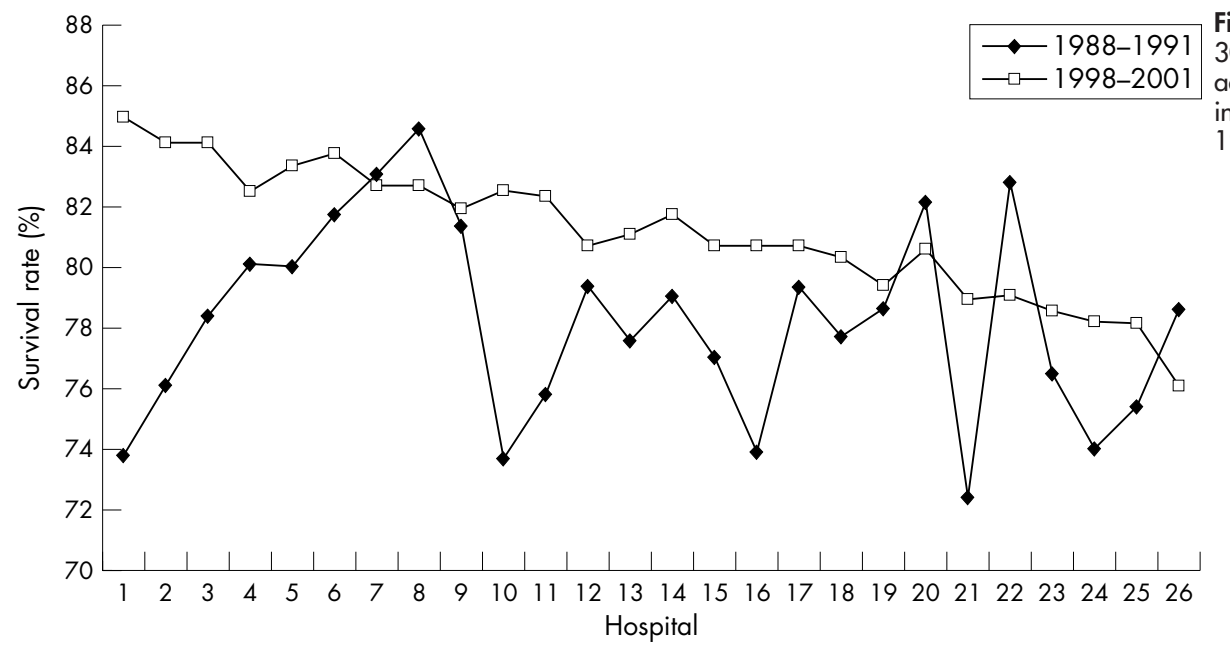

Figure 1 Age and sex standardised 30 day median survival rates after admission with acute myocardial infarction (AMI) between 1988 and 1991 and between 1998 and 2001. 


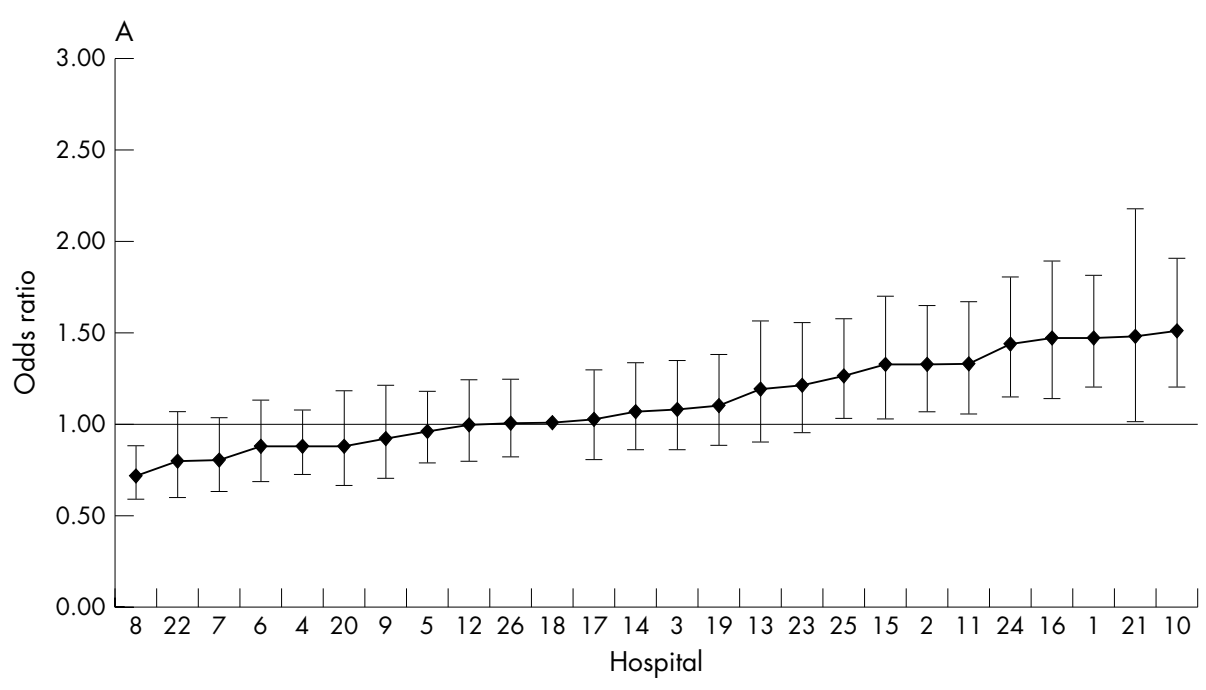

Figure 2 (A) Adjusted probability of death at 30 days after AMI between 1988 and 1991 . Odds ratios and $95 \%$ confidence intervals are shown; 18 is the index hospital. (B) Adjusted probability of death at 30 days after AMI between 1998 and 2001. Odds ratio and $95 \%$ confidence intervals are

shown; 9 is the index hospital.

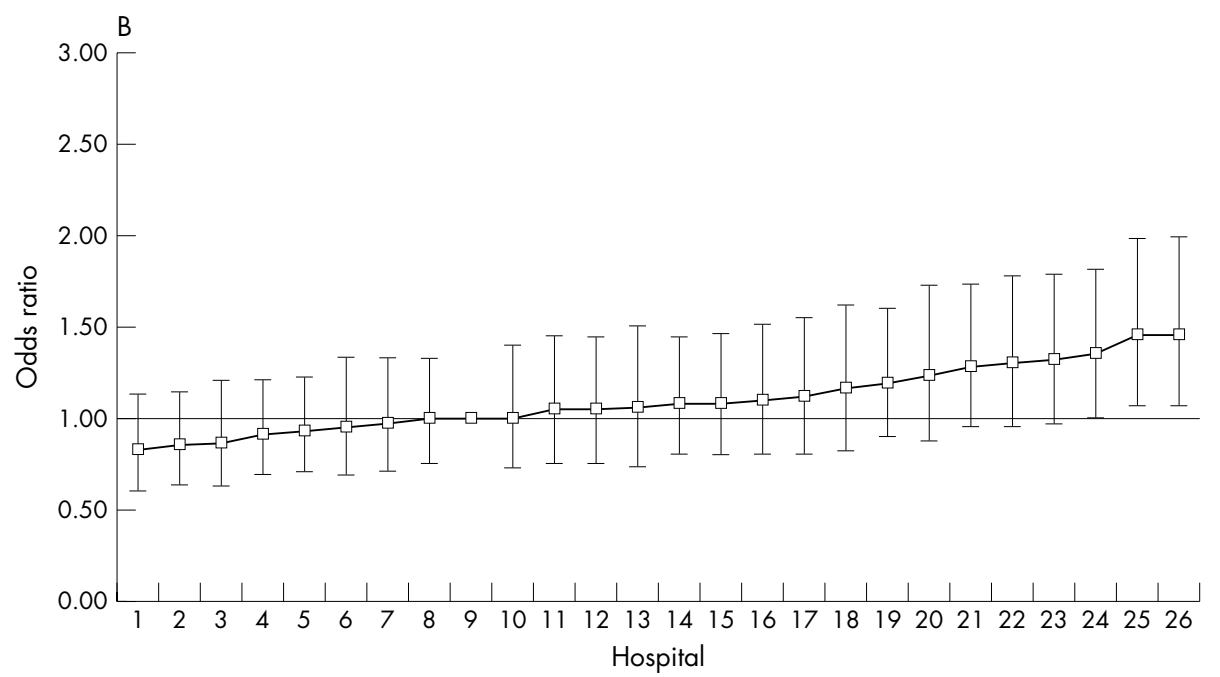

incidence of AMI between the two study periods, a finding consistent with prior studies from Scotland and elsewhere..$^{9-18}$ This may be attributable to a variety of factors including better primary prevention, falling rates of smoking, a change in the natural history of coronary artery disease, and better public and doctor awareness of the importance of potential cardiac symptoms. Similarly, the increase in 30 day survival after an AMI is also consistent with prior reports from Scotland and elsewhere. ${ }^{10-19}$ Several factors, including those that may have contributed to the decline in incidence, may be important. ${ }^{20}$ Others are changing thresholds for referral (whereby patients with chest pain are more likely to be referred to hospital by primary care practitioners) and admission (when referred patients are more likely than previously to be admitted). Diagnostic thresholds may also have been lowered, with consequent changes in severity of infarction. New treatments may also have played an important part.

The second major finding of this study (and the one most pertinent to the aim of the study) is that interhospital variation has declined substantially between 1988-1991 and 1998-2001. This is less easy to attribute to the factors mentioned above in connection with the decline in case fatality between these two periods. Indeed, our hypothesis was that greater input of cardiologists and reorganisation of care should have led to less between-hospital variation in 30 day survival and our findings are consistent with this (these factors may also, of course, have contributed to the improvement in survival).

The reduction in variation between hospitals seemed to be due, substantially, to a particularly notable improvement in survival in those hospitals that had the highest care fatality in the 1988-1991 study.

Though there was less variation between 1998 and 2001 than previously, interhospital variation persisted, with a nine percentage point difference in adjusted survival. The reasons for this require further study, as identification of correctable factors (such as deficiencies in treatment) may lead to further improvements in outcome and reductions in variation. This highlights the major limitation of this study, which is the absence of information on treatment and on other factors such as measurement of ventricular function, which may allow better adjustment of case mix.

In summary, the appointment of cardiologists and their involvement in the care of acute admissions to Scottish hospitals seem to be associated with an overall improvement (and less variation between hospitals) in survival after AMI.

\section{Authors' affiliations}

N F Murphy, S Stewart*, J J V McMurray, Department of Cardiology, Western Infirmary, Glasgow, UK

K Macintyre, Department of Public Health, University of Glasgow, Glasgow, UK 
S Capewell, Department of Public Health, University of Liverpool, Liverpool, UK

*Also the Division of Health Sciences, University of South Australia, Adelaide, Australia

Funding: NM is funded by the British Heart Foundation and SS is supported by the NH\&MRC of Australia and the NHF of Australia.

Ethical approval: Epidemiological and audit studies using anonymised routine datasets do not require specific ethics committee approval in Scotland.

\section{REFERENCES}

1 Capewell S, Kendrick S, Boyd J, et al. Measuring outcomes: one month survival after acute myocardial infarction in Scotland. Heart 1996;76:70-5.

2 Hanlon $P$, Beck S, Robertson $G$, et al. Coping with the inexorable rise in medical admissions: evaluating a radical reorganisation of acute medical care in a Scottish district general hospital. Health Bull (Edinb) 1997;55:176-84.

3 Kendrick S, Clarke J. The Scottish record linkage system. Health Bull (Edinb) 1993:51:72-9.

4 World Health Organization. Manual of the international statistical classification of disease, injuries and causes of death, 9th revision. Geneva: $\mathrm{WHO}, 1977$

5 Harley K, Jones C. Quality of Scottish Morbidity Record (SMR) data. Health Bull (Edinb) 1999:54:410-7.

6 NHS Scotland. Data quality assurance report on acute inpatient/day case data 2000-2002. January 2004:p17. http://www.isdscotland.org/isd/files/ SMRO1_National_Report.pdf.

7 Carstairs V, Morris R. Deprivation and health in Scotland. Aberdeen: Aberdeen University Press, 1991.

8 McLoone P. Carstairs scores for Scottish postcode sectors from the 2001 census. Glasgow: MRC Social \& Public Health Sciences Unit, March 2004 www.msoc-mrc.gla.ac.uk/Publications/pub/PDFs/Carstairs\%20Report/ Carstairs\%20report.pdf (accessed 24 November 2004).

9 Murphy NF, Maclntyre K, Capewell S, et al. Trends in hospital discharge rates for suspected acute coronary syndromes between 1990 and 2000: steep decline in acute myocardial infarction and marked increases in angina and other chest pain. BMJ 2004;328:1413-4.

10 Abildstrom SZ, Rasmussen S, Rosen $M$, et al. Trends in incidence and case fatality rates of acute myocardial infarction in Denmark and Sweden. Heart 2003:89:507-11.

11 Rosen M, Alfredsson L, Hammar N, et al. Attack rate, mortality and case fatality for acute myocardial infarction in Sweden during 1987-95: results from the national AMI register in Sweden. J Intern Med 2000;248:159-64.

12 Marques-Vidal P, Ruidavets JB, Cambou JP, et al. Incidence, recurrence, and case fatality rates for myocardial infarction in southwestern France, 1985 to 1993. Heart 2000;84:171-5.

13 Salomaa V, Miettinen H, Kuulasmaa K, et al. Decline of coronary heart disease mortality in Finland during 1983 to 1992: roles of incidence, recurrence, and case-fatality. The FINMONICA MI register study. Circulation 1996;94:3130-7

14 Roger VL, Jacobsen SJ, Weston SA, et al. Trends in the incidence and survival of patients with hospitalized myocardial infarction, Olmsted County, Minnesota, 1979 to 1994. Ann Intern Med 2002;136:341-8.

15 Rosamond WD, Chambless LE, Folsom AR, et al. Trends in the incidence of myocardial infarction and in mortality due to coronary heart disease, 1987 to 1994. N Engl J Med 1998:339:861-7.

16 Dauerman HL, Lessard D, Yarzebski J, et al. Ten-year trends in the incidence, treatment, and outcome of Q-wave myocardial infarction. Am J Cardiol 2000;86:730-5

17 Goldberg RJ, Yarzebski J, Lessard D, et al. A two-decades (1975 to 1995) long experience in the incidence, in-hospital and long-term case-fatality rates of acute myocardial infarction: a community-wide perspective. J Am Coll Cardiol 1999;33:1533-9.

18 Kostis JB, Wilson AC, Lacy CR, et al. Myocardial Infarction Data Acquistion System (MIDAS \#7) Study Group. Time trends in the occurrence and outcome of acute myocardial infarction and coronary heart disease death between 1986 and 1996 (a New Jersey statewide study). Am J Cardiol 2001;88:837-41.

19 Capewell S, Livingston BM, Maclntyre K, et al. Trends in case-fatality in 117 718 patients admitted with acute myocardial infarction in Scotland. Eur Heart $J$ 2000;21:1833-40.

20 Unal B, Critchley JA, Capewell S. Explaining the decline in coronary heart disease mortality in England and Wales between 1981 and 2000. Circulation 2004;109:1101-7.

\section{IMAGES IN CARDIOLOGY}

doi: $10.1136 /$ hrt.2004.044552 A paclitaxel coated stent used for in-stent restenosis within a sirolimus coated stent fails to protect against recurrent restenosis

$\mathrm{T}$ he optimal approach to in-stent restenosis (ISR) within a drug eluting stent (DES) remains uncertain. The Rotterdam research registry reported a zero major adverse cardiac event (MACE) rate at a median of 131 days in 10 patients treated with a paclitaxel coated Taxus DES for ISR within a sirolimus coated Cypher DES. However, this strategy may fail to prevent recurrent ISR. A 40 year old exsmoking white female with type 1 diabetes, renal failure, hypertension, hypercholesterolaemia, and a strong family history of premature coronary disease had inferior ischaemia on a myocardial perfusion scan and a $90 \%$ stenosis in the right coronary artery at angiography (panel A). This was predilated and stented with a $2.75 \times 33 \mathrm{~mm}$ Cypher (panel

B). Secondary prevention was optimised. Six months later angiography revealed ISR within the Cypher (panel C). This was predilated with a cutting balloon and stented with a 3.0 by $28 \mathrm{~mm}$ Taxus (panel D). Check angiography at eight months revealed very tight ISR in the Taxus (panel E). The implantation of a Taxus stent to treat ISR within a Cypher failed to prevent recurrent ISR.
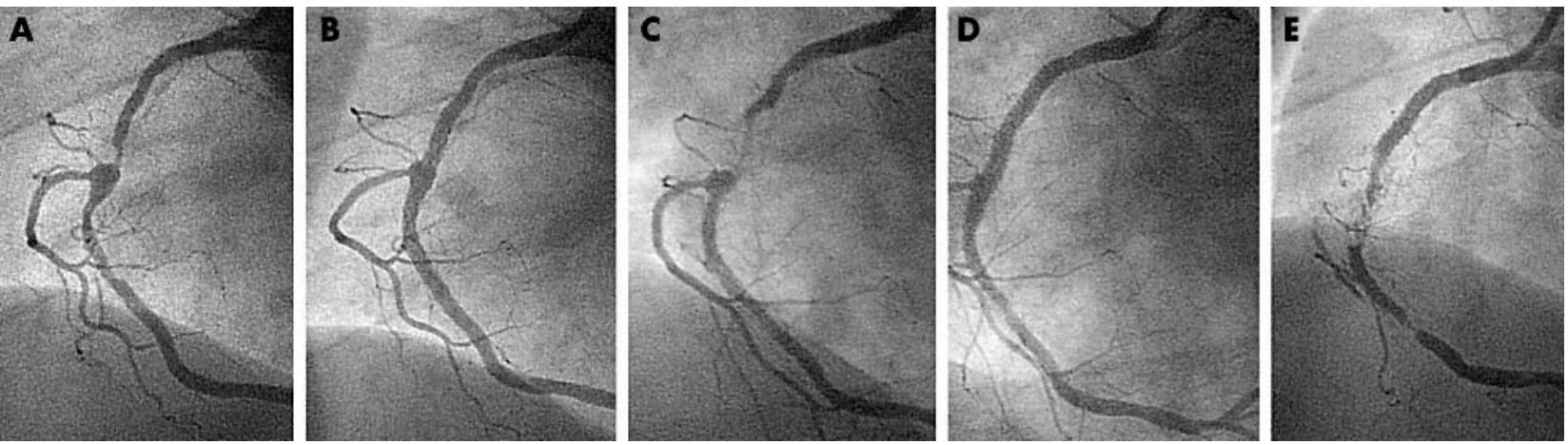